\title{
Optical Phase Conjugation for Dispersion and Nonlinearity Compensation in a 1600km, 42 Gb/s Quasi-Lossless System
}

\author{
P. Minzioni ${ }^{1}$, P. Harper ${ }^{2}$, V. Pusino ${ }^{1}$, L. Barker ${ }^{2}$, C. Langrock $^{3}$, M.M. Fejer ${ }^{3}$, J-D Ania-Castanon ${ }^{4}$, \\ I. Cristiani ${ }^{1}$, V. Degiorgio ${ }^{1}$ \\ 1. CNISM and Electronics Dept., University of Pavia, via Ferrata 1,I-27100 Pavia, Italy \\ 2. Photonics Research Group, Aston University, Birmingham, B4 7ET, U.K \\ 3. Edward Ginzton Laboratory, Stanford University, 450 Via Palou, 94305 Stanford, CA, USA \\ 4.Instituto de Óptica "Daza de Valdés”, CSIC, Madrid, Spain. \\ paolo.minzioni@unipv.it
}

\begin{abstract}
We experimentally investigate a long-distance, high-bit-rate transmission system which combines optical-phase-conjugation with quasi-lossless amplification. Comparison with a conventional system configuration demonstrates the possibility of obtaining both dispersion compensation and improved nonlinear tolerance using proposed scheme.

(C)2009 Optical Society of America

OCIS codes: (060.4510) Optical communications; (190.5040) Phase conjugation; (190.5650) Raman effect
\end{abstract}

\section{Introduction}

In high bit-rate communication links, optical nonlinearity along the link, and their interplay with the chromatic dispersion, is one of the major sources of signal distortion. In order to reduce the impact of fiber nonlinearity on signal transmission, an interesting possibility is given by the optical phase conjugation (OPC) technique. This technique is extremely promising, as, in principle it allows compensating both dispersive and nonlinear effects, if the third-order-dispersion effect (TOD) is negligible. This result can be obtained by simply inserting an OPC device in the middle of the link, as suggested by mid-span spectral-inversion (MSSI) technique [1]. Unfortunately MSSI cannot be generally used in installed transmission systems, as its effectiveness is strongly affected by the power asymmetries and fluctuations produced along the link by fiber losses. At the state of the art OPC can be effectively used only in specific configurations satisfying the mid-nonlinearity temporal-inversion (MNTI) requirements [2]. A different possibility that has recently been highlighted is that of using quasi-lossless (QL) transmission links, where the power fluctuations along the line are made negligible by the appropriate use of Raman distributed amplification [3], which closely approximate the ideal loss-less configuration required for MSSI implementation. Even if the use of the MNTI approach has proved to be effective for nonlinearity compensation [4], the evaluation of the MSSI approach in a QL system is particularly interesting as it mimics the ideal lossless situation. In this paper we show preliminary experimental results obtained by comparing the performance of an OPC-based QL-systems, with that of an OPC-based system employing lumped EDFA amplification, and of both EDFAs- and QL- systems in which the fiber dispersion is compensated by dispersion-compensating fiber (DCF), and not by OPC. This comparison, never done before, highlights both the separate and combined impact of OPC and QL-systems on transmission performance.

\section{Experimental setup}

The transmission experiments have been realized by means of a recirculating loop, which includes the transmission fiber, the amplification structure, and the OPC device as shown in Fig. 1. The transmitter, based on a pair of lithium niobate Mach-Zehnder modulators, produced a 42Gb/s RZ-OOK signal, with 33\% duty cycle at the signal wavelength $\lambda_{\mathrm{s}}=1548.8 \mathrm{~nm}$. This signal was periodically gated into the recirculating loop using an acousto-optic modulator (AOM) and optical coupler. The $80 \mathrm{~km} \mathrm{SMF-28} \mathrm{fibre} \mathrm{span} \mathrm{within} \mathrm{the} \mathrm{loop} \mathrm{was} \mathrm{enclosed} \mathrm{within} \mathrm{a} \mathrm{second-}$ order Raman-pumped cavity configured to provide quasi-lossless transmission along the entire span length [3]. After transmission through the span a portion of the circulating light was coupled from the loop into the OPC set up.

The OPC device is based on the cascading technique into a highly efficient, $67-\mathrm{mm}$ long, waveguide realized by reverse-proton-exchange on a periodically-poled lithium-niobate (PPLN) substrate. In order to achieve the polarization independence of the apparatus a polarization-diversity scheme like that reported in [5] has been used. By a proper implementation, high polarization independence can be obtained and the setup insertion losses, which for these devices are generally $>20 \mathrm{~dB}[6]$, are reduced below $9 \mathrm{~dB}$. The wavelength of the fundamental beam used for the second-harmonic generation (SHG) process has been set to $\lambda_{\mathrm{f}}=1552.52 \mathrm{~nm}$, and the crystal operating temperature to $108^{\circ} \mathrm{C}$, so as to avoid the photorefractive effect and to tune the SHG efficiency peak to $\lambda_{\mathrm{f}}$; the optical 
power output from the fundamental beam amplifier has been set to $250 \mathrm{~mW}$. This combination of signal and fundamental wavelengths gave a conjugate wavelength $\left(\lambda_{\mathrm{c}} \approx 2 \lambda_{\mathrm{f}}-\lambda_{\mathrm{s}}=1555.9 \mathrm{~nm}\right)$

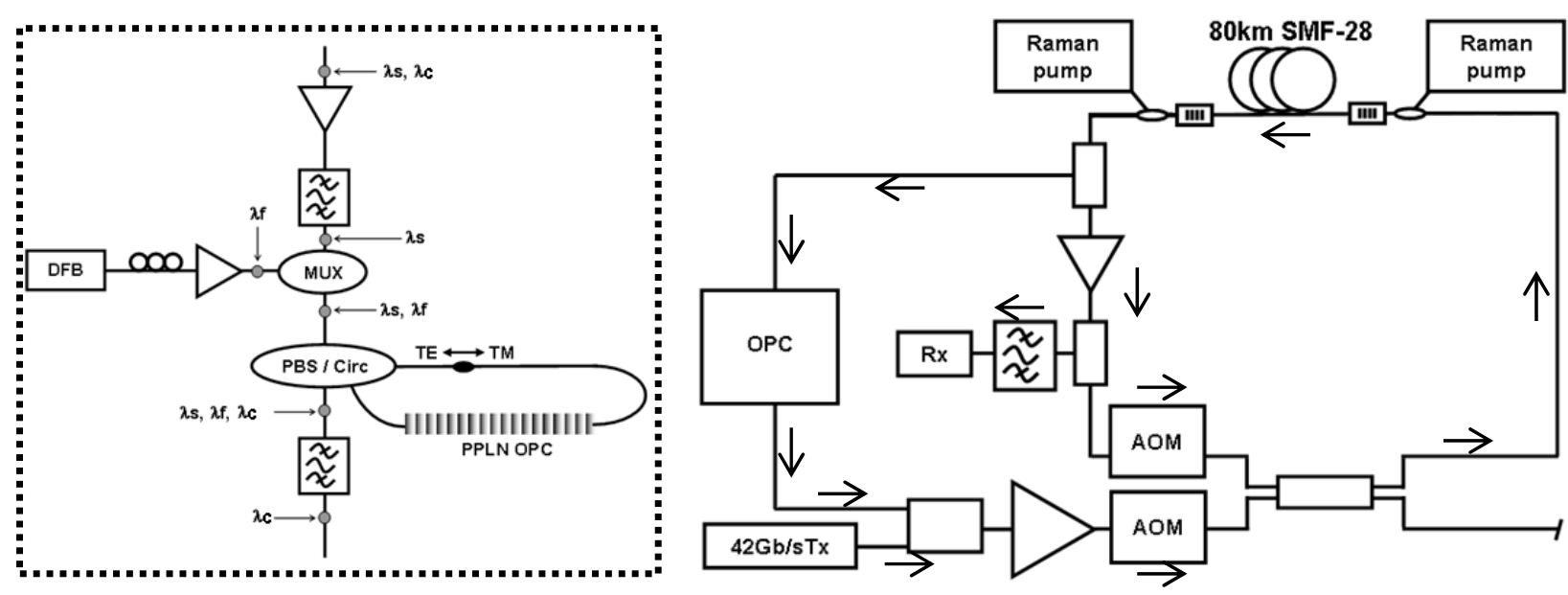

Fig. 1. Recirculating loop configuration and (insert) OPC set-up.

In order to perform comparison experiments without the OPC, and by using DCF to compensate fiber dispersion, the OPC setup has been removed from the loop, and appropriate DCF modules have been inserted. Further comparison experiments of a conventional lumped amplification configuration were done both with and without the $\mathrm{OPC}$ by inserting an additional EDFA to replace the quasi-lossless amplification scheme.

\section{Experimental results and discussion}

In order to compare the performance of such different transmission systems we evaluated for each linkconfiguration the launch-power tolerance. This has been done by measuring, after transmission on a $12 \times 80 \mathrm{~km}$ link, the Q-factor penalty (with respect to the best achievable BER performance) as a function of the optical power launched into the propagation spans. This graph allows showing the impact on the different transmission system of a non-ideal power setting. In Fig.2, we compare the power-tolerance of EDFA- and QL- systems when dispersion compensation is achieved by means of DCF (left side) and OPC (right side).
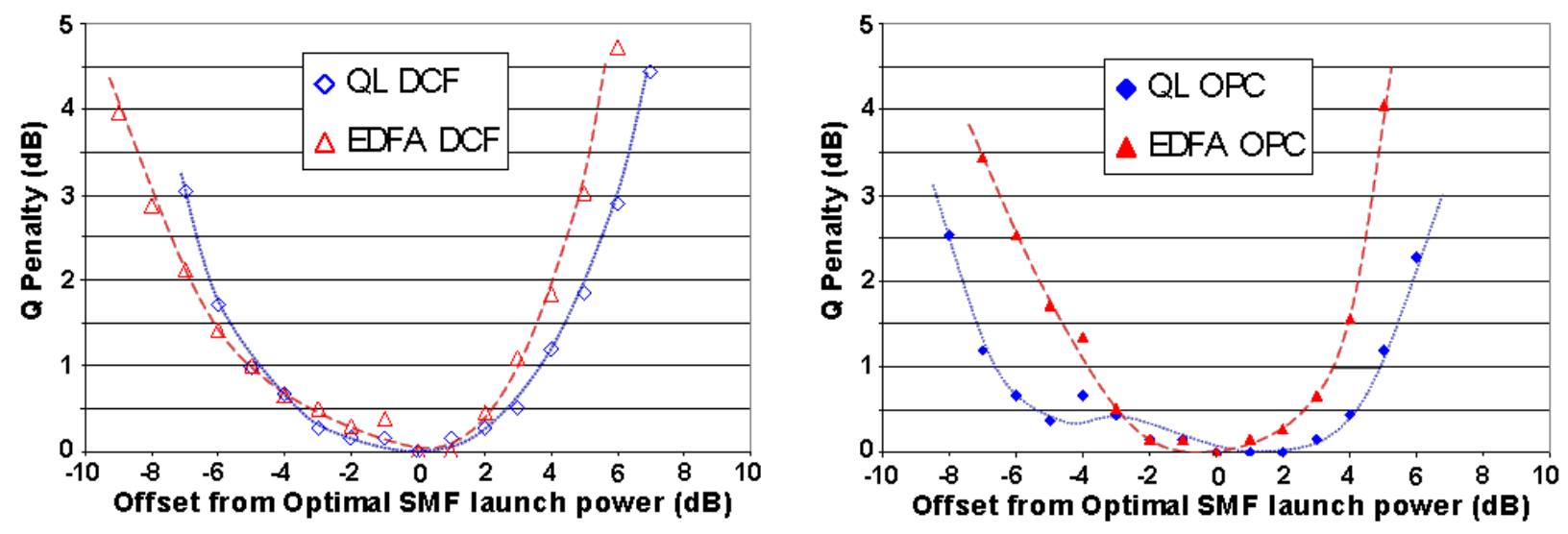

Fig. 2. Comparison of Q-penalty as a function of the optical power launched in the fiber spans for EDFA- and QL- links when Left: dispersion compensation is obtained by DCF, Right: dispersion compensation is obtained by OPC.

It can be seen that, when DCF is used, changing the amplification scheme do not yield an evident improvement of the system tolerance; this result is due to the fact that, in this case, no nonlinearity can be compensated, independently of the amplification structure. Conversely the situation changes significantly when OPC is employed: when OPC and lumped amplification is used, MSSI implementation doesn't allow nonlinearity compensation and the obtained tolerance (at a 1-dB penalty-level) is about 7.0 dB; instead when the QL-system is used OPC allows compensating the slight nonlinear distortions affecting pulse propagation, and a power tolerance in excess of 
$11.5 \mathrm{~dB}$ is obtained. Due to the constant power level along the transmission fibre in the QL case the optimal launch power of $\sim-5 \mathrm{dBm}$ was considerably lower than $+2 \mathrm{dBm}$ level when the EDFA was used.

In order to complete the analysis, we have also directly compared the QL-DCF and QL-OPC system tolerances and performances in Fig. 3. The graph on the left shows the improvement, as could be expected by previous considerations, on launch-power tolerance given by the QL-OPC system. The graph on the right instead shows the best BER obtained by each of the two system configurations as a function of the propagation distance. It can be observed that, when the propagation distance is short, the OPC-based system-performance is worse than that obtained by DCF-based one. Conversely, for distances above $1000 \mathrm{~km}$ the BER obtained by using OPC is better than that produced using DCF, because of the combination of two effects. The first one is that, even if the impact of fiber nonlinearity is modest (both the launch power and the nonlinear coefficient of the SSMF are low) a small nonlinear distortion can accumulate during propagation on the QL-DCF system, while any nonlinear distortion should be compensated on the QL-OPC one. The second effect is related to the ASE noise accumulation. In the QLOPC system in fact the OPC setup introduces a OSNR degradation, once along the system. Conversely, by using DCF, a very small OSNR reduction is produced by the optical amplifiers used to compensate for DCF attenuation, but this degradation is repeated once in every span, and so for long propagation distances they can sum up and become relevant on the evaluation of system performance.

It must be underlined, by looking at the short-distance results, that the measured performance for the OPC-link is affected by some small problem, probably related to the ASE noise added by the high-power amplifiers in the OPCsetup. The OPC-setup is currently being optimized to solve this problem; hence we believe that the performance obtained by the QL-OPC systems can still be significantly improved.
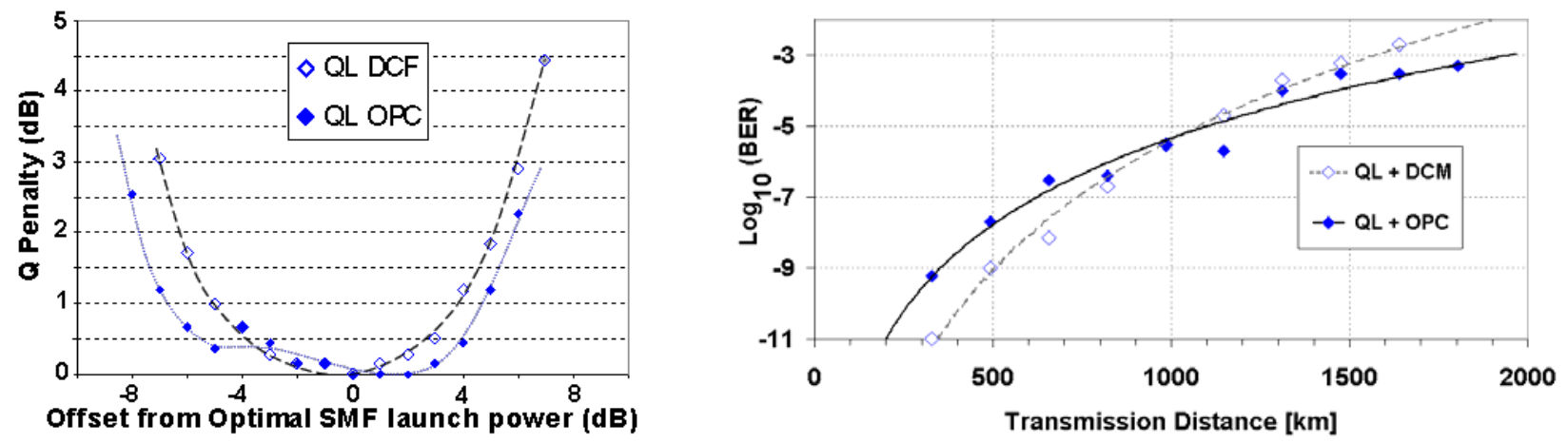

Fig. 3. On the left: comparison on launch-power tolerance between QL- links using DCFs and OPC for dispersion compensation. On the right: BER curve as a function of the system length obtained considering the QL+DCf and QL+OPC system configurations.

\section{Conclusions}

We show, for the first time, the advantages given by the use of the OPC technique in the transmission of a $42 \mathrm{~Gb} / \mathrm{s}$ RZ-ASK signal along a 1600km QL-system. The use of OPC in QL-system is extremely interesting as it provides a basis for studying MSSI with close to the ideal lossless, symmetric power profile. Our results show that, in absolute terms, OPC offers a better performance than DCF for long transmission distances, attributable to a reduction of the noise accumulation. In addition, the combination of QL+OPC offers much improved launch power tolerance when compared to any other of the considered alternatives, thanks to the minimization of nonlinear penalties. We conclude that the use of OPC in QL-systems is extremely interesting as it allows for the removal of DCF spools (and related amplifiers), and allows simultaneous compensation of both dispersive and nonlinear impairments. Further experiments to highlight the nonlinearity compensation effect are currently planned and will be performed soon.

\section{References}

[1] A. Yariv et al, "Compensation for channel dispersion by nonlinear optical phase conjugation" Opt. Lett. 4, $52-54$ (1979)

[2] P. Minzioni et al., "Unifying theory of compensation techniques for intrachannel nonlinear effects" Opt. Express 13, 8460-8468 (2005)

[3] J.D. Ania-Castañón et. al "Simultaneous spatial and spectral transparency in ultra-long fiber lasers" Phys. Rev. Lett. 101(2008), 123903

[4] P. Minzioni et al., "Experimental Demonstration of Nonlinearity and Dispersion Compensation in an Embedded Link by Optical Phase Conjugation" IEEE Photon. Technol. Lett. 18, 995-997 (2006)

[5] V. Pusino et al, "Wavelength Conversion of Real-Time 100-Gb/s POLMUX RZ-DQPSK”. OFC 2009, Presentation OThS3 (2009)

[6] S.L. Jansen et al, “16 40 Gb/s Over 800 km of SSMF Using Mid-Link Spectral Inversion” IEEE Photon. Technol. Lett. 16, 1763-1765 (2004) 